\title{
Eficiência reprodutiva e influência de fatores de meio e de herança sobre a variação no peso ao nascer de bubalinos no estado de Rondônia
}

\section{Chiara Rodrigues de Amorim Lopes ${ }^{1}$, Severino Benone Paes Barbosa ${ }^{2}$, Ricardo Gomes de Araújo Pereira ${ }^{3}$, Kleber Régis Santoro ${ }^{4}$, Aguirres Valongo de Lira $^{5}$}

\footnotetext{
${ }^{1}$ Doutoranda em Produção Animal/UFRPE.

2 DZ/UFRPE.

${ }^{3}$ Embrapa/CPAFRO/EMBRAPA-RO.

${ }^{4}$ DZ/GARANHUS.

${ }^{5}$ Mestrando em Produção Animal/UFRPE.
}

RESUMO - Avaliaram-se as características reprodutivas e o peso ao nascer em bubalinos mestiços e determinaramse os possíveis fatores de sua variação. Utilizaram-se 115 informações de idade ao primeiro parto e 420 intervalos de parto referentes a 127 fêmeas 1/2, 3/4, 7/8, 15/16 Murrah × Mediterrâneo e puras por cruza (PC). Também foram utilizados 557 pesos ao nascer de progênies resultantes do cruzamento entre essas fêmeas e cinco touros da raça Murrah. Os nascimentos ocorreram no período de 1979 a 2001. A idade ao primeiro parto $(1291 \pm 235$ dias $)$ foi influenciada pelo grupo de contemporâneos da búfala. O intervalo de partos (451 \pm 139 dias) foi afetado pelos efeitos fixos grupo de contemporâneos da búfala; peso da búfala no pós-parto anterior; idade da búfala ao parto anterior, linear e quadrática; bem como, pelo efeito aleatório de búfala. O grupo genético não determinou variações sobre as características idade ao primeiro parto e intervalo de partos. O peso ao nascer $(34,25 \pm 5,02 \mathrm{~kg})$ foi influenciado pelo sexo da cria, pelo grupo de contemporâneos do bezerro e pelo peso da búfala após o parto, além do efeito aleatório de mãe. O grupo genético da búfala, a idade da búfala ao parto e o pai da cria não influenciaram o peso ao nascer. A herdabilidade estimada do peso ao nascimento foi de 0,60 $\pm 0,12$. Os fatores que apresentaram diferenças significativas devem ser considerados na avaliação das características reprodutivas $e$ do peso ao nascer.

Palavras-chave: Bubalus bubalis, desempenho reprodutivo, herdabilidade, idade ao primeiro parto, intervalo de partos

\section{Reproductive performance, genetic and environmental effects on birth weight of Buffaloes in Rondonia State}

\begin{abstract}
The objectives of this study were to evaluate reproductive traits (age at first calving and calving interval) and to obtain heritability estimates for birth weight in a crossbred (Murrah and Mediterranean breeds) Buffalo population in Rondonia State, Brazil. Data recorded between 1979 and 2001 were analyzed by SAS ${ }^{\circledR}$. Variance components and heritability estimates were obtained by using the DFREML program. Age at first calving $(1,291 \pm 235$ days) was significantly influenced by the contemporary group of dam. Calving interval (451 \pm 139 days) was significantly influenced by the fixed effects of contemporary group of dam, weight of dam at previous calving, linear and quadratic components of age of dam at previous calving and the random effect of dam. Birth weight (34.25 $\pm 5.02 \mathrm{~kg})$ was influenced by the fixed effects of sex, contemporary group of calf, weight of dam at calving and by the random effect of dam. Heritability estimate for birth weight was high $(0.60 \pm 0.12)$, suggesting genetic gains may be obtained by selection for birth weight in this Buffalo population.
\end{abstract}

Key Words: age at first calving, Bubalus bubalis, calving interval, heritability, reproductive performance

\section{Introdução}

Os búfalos são animais produtivos e bastante adaptáveis às diversas condições ambientais, por isso, sua exploração encontra-se em ampla expansão na pecuária nacional. De acordo com a FAOSTAT (2005), o rebanho brasileiro possui mais de 1,2 milhões de cabeças, cujo maior volume se encontra na Região Norte.

A reprodução e a produção são eventos fisiológicos determinantes da rentabilidade econômica da pecuária, tanto de corte como leiteira. Entre as características reprodutivas que mais afetam a produtividade de um 
rebanho, a idade ao primeiro parto e o intervalo de partos são as mais relevantes (Ramos et al., 2004). De acordo com Pereira (2004), o intervalo de partos é um dos principais parâmetros para medir a eficiência reprodutiva de um rebanho, pois, quanto menor, maior será o número de crias produzidas por animal durante sua vida útil.

Neste sentido, Téllez et al. (2005) reportam que intervalo de partos de 12 meses é fisiologicamente possível e economicamente vantajoso. Baruselli \& Henriquez (2002) consideram aceitável que a búfala produza dois bezerros a cada três anos. A idade ao primeiro parto também se destaca nas relações entre características produtivas e reprodutivas de um rebanho. Búfalas precoces à puberdade produzem mais crias e leite em sua vida produtiva.

De acordo com Costa (1999) e Tonhati et al. (2000), as características de fertilidade apresentam herdabilidade muito baixa e são influenciadas pelos efeitos ambientais. Entretanto, Cassiano et al. (2004) afirmam que a importância das características reprodutivas em programas de melhoramento genético está relacionada, principalmente, às taxas de ganho genético anual.

Segundo Tonhati et al. (1997), a diminuição da idade ao primeiro parto reduz o intervalo de gerações e aumenta a vida produtiva e a intensidade de seleção nas fêmeas. Esses autores reportam que a diminuição do intervalo de partos induz ao aumento de produção na vida útil da búfala e reflete positivamente no progresso genético do rebanho. Considerando esses fatores, Pereira (2004) aponta o atraso genético quantificado pelos indicadores reprodutivos como o principal desafio a ser superado no melhoramento genético dos rebanhos.

Em bubalinos, assim como nas demais espécies animais, o peso ao nascer constitui característica produtiva de elevada importância zootécnica, em virtude de sua relação com a taxa de sobrevivência ao desmame e com o peso nas demais fases de desenvolvimento do animal. Segundo Nogueira et al. (1997), bezerros bubalinos que apresentam maiores pesos ao nascimento tendem a ganhar peso mais rapidamente antes e depois do desmame. Segundo Pereira (2004), é uma característica de ampla variação, o que torna necessário medir o quanto dessa variação é de natureza genética e o quanto é de natureza ambiental.

O peso ao nascer é também uma das primeiras expressões fenotípicas do genótipo individual e pode ser utilizado como auxílio na seleção precoce de animais em programas de melhoramento genético animal. Nesse sentido, o conhecimento da herdabilidade é fundamental para definição do esquema de seleção mais adequado.

Os valores de herdabilidade do peso ao nascer encontrados na literatura variam de 0,10 a 0,62 (Balieiro et al., 1996;
Cassiano et al., 2004), o que sugere a necessidade de mais estudos sobre esta característica para definição do quanto é influenciada pelo manejo e do quanto é influenciada pelos aspectos genéticos.

Objetivou-se neste estudo avaliar da idade ao primeiro parto e o intervalo de partos de búfalas mestiças das raças Murrah e Mediterrânea e estudar os efeitos de fontes nãogenéticas que podem causar variação destas características. Avaliaram-se ainda os fatores de meio e de herança como fontes de variação no peso ao nascer de bezerros bubalinos mestiços criados em sistema de manejo extensivo no estado de Rondônia.

\section{Material e Métodos}

Foram utilizadas 115 informações de idade ao primeiro parto e 420 intervalos de partos provenientes de 127 fêmeas de composições raciais 1/2, 3/4, 7/8, 15/16 Murrah $\times$ Mediterrâneo e puros por cruza (PC). Também foram utilizados 557 pesos ao nascer de progênies resultantes do cruzamento entre essas fêmeas e cinco touros da raça Murrah. Os nascimentos ocorreram no período de 1979 a 2001. Os dados foram coletados em um sistema de produção do Centro Agroflorestral da Embrapa de Rondônia (CPAFRO/ EMBRAPA - RO), localizado na cidade de Porto Velho.

Segundo classificação de Köppen, o estado de Rondônia apresenta clima tropical chuvoso, do tipo Am, que se caracteriza por total pluviométrico anual elevado e curto período de estiagem. O município de Porto Velho abrange uma área de $35.928,9$ km², cujas coordenadas geográficas são 876' Sul e $63^{\circ} 08^{\prime}$ Oeste e altitude de $95 \mathrm{~m}$. O clima desta região é equatorial quente e úmido, com estiagem no período de junho a setembro e índice pluviométrico que pode chegar a $2.000 \mathrm{~mm} / \mathrm{ano}$. De acordo com dados meteorológicos observados pelo Instituto Nacional de Meteorologia (INMET, 2006), as temperaturas médias, máximas e mínimas oscilam entre 24 e $26^{\circ} \mathrm{C}$; 28 e $33^{\circ} \mathrm{C}$; e 18 e $21^{\circ} \mathrm{C}$, respectivamente, e as médias anuais de umidade relativa do ar oscilam entre $80 \mathrm{e}$ 90\%. A insolação e evaporação anuais são de aproximadamente 1.770 horas e acima de $750 \mathrm{~mm}$, respectivamente.

Os animais foram criados extensivamente em pastagens de Brachiaria humidicola (Quicuio da amazônia) até 1987. Em 1988, foram implantados no sistema Andropogon gayanus, Brachiaria brizantha, cv. Marandu, e Pueraria phaseolóides, além de uma capineira de Cameron (Pennisetum purpureum) para suplementação alimentar no período seco. Todo o rebanho recebia mistura mineral à vontade.

A ordenha era realizada uma vez ao dia e o controle leiteiro era feito quinzenalmente. Os bezerros permaneciam 
com as mães até 6 a 7 meses de idade, quando eram desmamados. A taxa de substituição de fêmeas adultas era de $10 \%$ ao ano.

O sistema de acasalamento utilizado era o de monta natural, a campo, na proporção de um touro para 40 búfalas. Fêmeas a partir de 350 kg eram consideradas aptas à reprodução e permaneciam em lotes contendo um touro até dois meses antes da parição, quando eram transferidas para piquetes de maternidade.

Após a parição, procedeu-se ao corte e à desinfecção do umbigo. Depois da primeira mamada do colostro, mãe e crias eram pesadas e submetidas às práticas sanitárias e vacinações e ao controle de endo e ectoparasitos, sistematicamente. Foram definidas, a partir dos dados climatológicos do INMET, duas épocas de parto: seca (junho-setembro) e chuvosa (outubro-maio). Posteriormente, foram definidos grupos de contemporâneos de ano-época de nascimento da búfala para idade ao primeiro parto, de ano-época de ocorrência do parto precedente para intervalo de partos, e de grupos de ano-época de nascimento do bezerro para peso ao nascer.

A idade ao primeiro parto foi analisada utilizando-se um modelo estatístico ajustado considerando o grupo genético da búfala $(1=1 / 2 \mathrm{Mu} ; 2=3 / 4 \mathrm{Mu} ; 3=7 / 8 \mathrm{Mu} ; 4=$ 15/16 Mu; 5 = PC Mu) e o grupo de contemporâneos da búfala (ano-época de nascimento da búfala). O intervalo de partos foi analisado por meio de um modelo estatístico ajustado considerando o grupo genético da búfala ( 1 = $1 / 2 \mathrm{Mu} ; 2$ = 3/4 Mu; 3 = 7/8 Mu; 4 = 15/16 Mu), o grupo de contemporâneos da búfala (ano-época de ocorrência do parto precedente), as co-variáveis peso da búfala após o parto anterior e a idade da búfala ao parto anterior (nos termos linear e quadrático) e, como efeito aleatório, a búfala. Neste modelo testaram-se diversas estruturas da matriz de co-variância residual objetivando determinar a que melhor se ajustava aos dados. A seleção da estrutura da matriz de co-variância residual foi feita por meio do critério de informação de Akaike (AIC), segundo recomendações de Wolfinger (1993).

Para análise do peso ao nascer, ajustou-se um modelo com os efeitos fixos de grupo genético da búfala $(1=1 / 2 \mathrm{Mu}$; $2=3 / 4 \mathrm{Mu} ; 3=7 / 8 \mathrm{Mu} ; 4=15 / 16 \mathrm{Mu}$ ), sexo do bezerro e grupo de contemporâneos (ano-época de nascimento do bezerro), as co-variáveis idade ao parto e peso da búfala ao parto (termo linear) e os efeitos aleatórios de mãe e pai do bezerro.

$\mathrm{Na}$ análise de regressão do intervalo de partos, foram considerados o peso da búfala pós-parto anterior e a idade da búfala no parto anterior e, na análise de regressão, o peso ao nascer e o peso da búfala pós-parto. Na análise da idade ao primeiro parto, utilizou-se o procedimento PROC
GLM (SAS, 2000). Para as análises de intervalo de partos e do peso ao nascer, utilizou-se a metodologia dos modelos lineares mistos, com máxima verossimilhança restrita, por meio do procedimento PROC MIXED (SAS, 2000).

A estimativa dos componentes de variância e da herdabilidade para peso ao nascer foram feitas pelo programa DFREML 3.1 (Meyer, 2000) utilizando-se o método de máxima verossimilhança restrita livre de derivadas. O critério de convergência adotado foi de $10^{-6}$ e o modelo misto continha os efeitos fixos grupo genético da búfala $(1=1 / 2 \mathrm{Mu} ; 2=3 / 4 \mathrm{Mu} ; 3$ = 7/8 Mu; $4=15 / 16 \mathrm{Mu})$, sexo do bezerro e grupo de contemporâneos (ano-época de nascimento do bezerro), as co-variáveis idade ao parto e peso da búfala ao parto (termo linear) e os efeitos aleatórios de mãe e pai do bezerro.

\section{Resultados e Discussão}

As médias da idade ao primeiro parto e do intervalo de partos foram de $1291 \pm 235$ e $451 \pm 139$ dias, respectivamente (Tabela 1). Para estudo da idade ao primeiro parto, foram gerados no modelo um coeficiente de determinação de $61 \%$ e um coeficiente de variação de $13 \%$, indicando que parte das fontes que causam variação na característica é explicada pelo modelo utilizado.

O grupo genético da búfala não influenciou $(\mathrm{P}>0,05)$ a idade ao primeiro parto (Tabela 1). Possivelmente, o tipo de manejo adotado, principalmente alimentar, não permitiu às búfalas expressarem seus desempenhos de idade ao primeiro parto.

O grupo de contemporâneos da búfala (ano-época de nascimento da búfala) influenciou significativamente $(\mathrm{P}<0,01)$ a idade da búfala ao primeiro parto (Tabela 1$)$, no entanto, esta característica não apresentou tendência de redução ou aumento no período estudado e variou aleatoriamente entre grupos de contemporâneos, fato que pode estar relacionado ao modo de criação, totalmente extensivo, dos animais, uma vez que mudanças nas condições climáticas de um ano a outro influenciaram o desempenho reprodutivo das fêmeas.

Sampaio Neto et al. (2001) verificaram também que o ano de nascimento da búfala ocasionou variações significativas na idade ao primeiro parto. Entretanto, esses autores verificaram redução, ano a ano, da idade ao primeiro parto e atribuíram essa tendência à adoção de técnicas de manejo eficientes e à seleção para animais mais precoces durante determinado período. Silva et al. (1995), avaliando um rebanho bubalino criado em sistema de pastejo contínuo na região nordeste do Paraná, também verificaram influência do ano de nascimento da búfala sobre a idade ao primeiro 
Tabela 1 - Resumo da análise de variância para idade ao primeiro parto e intervalo de partos

\begin{tabular}{lcccc}
\hline & \multicolumn{2}{c}{ Idade ao primeiro parto } & \multicolumn{2}{c}{ Intervalo de partos } \\
\cline { 2 - 5 } Fonte de variação & GL & Valor de F & GL & 3 \\
\hline Grupo genético da búfala & 4 & $0,72^{\text {ns }}$ & 32 & $1,30^{\text {ns }}$ \\
Grupo de contemporâneos da búfala & 24 & $4,67^{* *}$ & 1 & $1,63^{* *}$ \\
Peso da búfala após o parto & - & - & 1 & $10,22^{* *}$ \\
Idade ao parto anterior linear & - & - & 1 & $9,12^{* *}$ \\
Idade ao parto anterior quadrática & - & - & $9,28^{* *}$ \\
\hline
\end{tabular}

** $=\mathrm{P}<0,01 ; \mathrm{ns}=\mathrm{P}>0,05$.

parto. Considerando esse resultado, os autores sugeriram que a idade ao primeiro parto pode ser antecipada por meio de melhorias na nutrição e no manejo dos animais.

Para realização da análise de intervalo de partos, a estrutura de co-variância que melhor se ajustou aos dados, com base no critério de informação de Akaike (AIC), foi a autoregressiva heterogênea. O grupo genético da búfala não influenciou $(\mathrm{P}>0,05)$ o intervalo de partos (Tabela 1 ), possivelmente em virtude das condições ambientais, que não permitiram aos animais expressarem seu potencial genético para redução, em dias, do intervalo de partos.

O grupo de contemporâneos da búfala ao parto anterior foi significativo $(\mathrm{P}<0,01)$ na avaliação do intervalo de partos (Tabela 1). No entanto, o intervalo de partos não apresentou tendência de redução ou aumento durante o período avaliado.

Possíveis diferenças climáticas e de disponibilidade de pasto entre ano e época devem ter proporcionado à fêmea recém-parida recuperação mais rápida ou tardia de suas reservas corporais e, seguidamente, de sua atividade ovariana. A conseqüência é menor ou maior período do parto até a nova concepção, o que está diretamente relacionado ao intervalo até o próximo parto. Resultados nesse sentido foram verificados por Aziz et al. (2001) no Egito. Esses autores observaram que a estação e o ano do parto tiveram efeitos significativos sobre o intervalo de partos de búfalas recebendo suplementação com concentrado de acordo com a produção leiteira. Em avaliação de búfalas sob dois sistemas de manejo, também no Egito, Abdalla (2003) concluiu que o efeito sobre a inibição da atividade ovariana é menor quando os animais são criados em confinamento com acesso a pastagem e na presença contínua do reprodutor. Em estudos realizados por Sampaio Neto et al. (2001), o mês do parto anterior de búfalas mantidas em sistema intensivo de criação consistiu em fonte de variação significativa para esta característica. Esses autores concluíram que búfalas parindo no período chuvoso apresentaram menor intervalo de partos, logo, a maior disponibilidade de forragem nesse período reduz o estresse produtivo nos animais e possibilita a redução do intervalo de partos.

Observou-se efeito significativo $(\mathrm{P}<0,01)$ do peso pós-parto anterior sobre o intervalo de partos (Tabela 1).
Provavelmente, búfalas com maior peso após o parto anterior tiveram condições para recuperação de suas reservas corporais em menor tempo, além de uma ativação mais rápida da atividade ovariana e menor intervalo de parto. Esses resultados confirmam estudos realizados por Shah (1991), no Paquistão. Esse autor observou que a maior perda de peso após o parto diminuiu a probabilidade de um recomeço do ciclo ovariano até 45 dias após o parto. Ribeiro et al. (1997) observaram que o escore da condição corporal de búfalas mestiças na região do Baixo Amazonas afetou o intervalo do parto ao primeiro cio observado. Certamente, quanto maior o intervalo do parto ao aparecimento do primeiro cio, maior a probabilidade de aumento do intervalo de partos e redução do desempenho reprodutivo, de modo geral.

Verificou-se efeito quadrático significativo $(\mathrm{P}<0,01)$ da idade da búfala ao parto anterior sobre o intervalo de partos (Tabela 1). A partir da derivação da equação de regressão, observou-se que menores intervalos de partos podem ser obtidos em búfalas com 10 anos de idade. Em estudo realizado por Cassiano et al. (2003), a ordem de parto influenciou significativamente o intervalo de partos nos estudos. Esses autores verificaram que búfalas atingem melhor desempenho da quinta à sétima parição, pois, neste período, além de produzirem bezerros maiores, apresentam intervalos de partos mais curtos.

Pela análise de regressão, verificou-se que o intervalo de partos pode ser estimado pela associação entre o peso da búfala pós-parto precedente e a idade da búfala ao parto anterior, linear e quadrática. A equação de regressão ajustada pelo modelo que explica variações significativas no intervalo de partos foi:

$\hat{\mathrm{Y}}=774,8482959-0,2205727 \mathrm{X}-0,1319681 \mathrm{Z}+0,0000175 Z^{2}$

em que: $\hat{Y}$ = intervalo de partos estimado; $X$ = peso, em $\mathrm{kg}$, da búfala pós-parto precedente; $\mathrm{Z}$ = idade, em dias, da búfala ao parto anterior.

$\mathrm{O}$ peso ao nascer de bezerros bubalinos apresentou valor médio e desvio-padrão de 34,20 \pm 5,02 kg (Tabela 2).

O grupo genético da búfala não foi considerado importante fonte de variação $(P>0,05)$ sobre o peso ao nascer de 
bubalinos (Tabela 2). Cassiano et al. (2003), avaliando a importância do grupo genético de machos e fêmeas sobre o peso ao nascer em bubalinos, progênies de animais puros e 1/2 Jafarabadi, Murrah, Carabao, Mediterrâneo e Baio, verificaram que apenas o grupo genético dos machos determinou variação sobre a característica produtiva, uma vez que os reprodutores do tipo Baio apresentaram proles mais pesadas ao nascimento.

O sexo do bezerro constitui importante fonte de variação $(\mathrm{P}<0,01)$ e afeta o peso ao nascer (Tabela 2$)$. O peso médio dos bezerros machos ao nascimento $(34,16 \pm 4,41)$ foi superior ao peso médio das fêmeas $(32,93 \pm 4,97)$. Esses resultados são similares aos encontrados por Baruselli (1993), Balieiro et al. (1996) e Das et al. (2004), que observaram maiores pesos médios observados nos bezerros machos.

O grupo de contemporâneos teve influência significativa $(\mathrm{P}<0,01)$ sobre o peso ao nascer de bezerros bubalinos (Tabela 2). No entanto, as variações desta característica foram aleatórias ao longo dos anos e não obedeceram a uma ordem crescente ou decrescente, o que provavelmente reflete o sistema extensivo de criação praticado no Centro de Pesquisa, no qual possíveis diferenças climáticas e de disponibilidade de pasto interferiram diretamente nas condições nutricionais e fisiológicas dos animais, causando maiores impactos nas fêmeas ao terço final da gestação e, conseqüentemente, no peso inicial dos bezerros, uma vez que é no terço final da gestação que ocorre maior desenvolvimento fetal, portanto, maior exigência nutricional. Yadav et al. (2001), estudando nove diferentes períodos, também observaram forte influência desta variável sobre o peso ao nascer.

A idade das fêmeas, como efeito linear, não influenciou $(\mathrm{P}>0,05)$ o peso da cria ao nascimento (Tabela 2). Por outro lado, o peso da matriz pós-parto foi considerado importante causa de variação e teve influência significativa $(\mathrm{P}<0,01)$ sobre o peso inicial dos bezerros (Tabela 2).

Por meio da análise de regressão, verificou-se que o peso ao nascer de bezerros bubalinos pode ser estimado considerando o peso da búfala pós-parto. A equação de regressão ajustada pelo modelo que explica variações significativas peso ao nascer foi:

$$
\hat{\mathrm{Y}}=24,09801517+0,01861442 \mathrm{X}
$$

Tabela 2 - Resumo da análise de variância para o peso ao nascer

\begin{tabular}{lcc}
\hline Fonte de variação & GL & Valor de F \\
\hline Grupo genético da búfala & 3 & $0,90^{\mathrm{ns}}$ \\
Sexo do bezerro & 1 & $6,56^{*}$ \\
Grupo de contemporâneos do bezerro & 41 & $2,50^{* *}$ \\
Idade da búfala ao parto & 1 & $3,60^{\mathrm{ns}}$ \\
Peso da búfala após o parto & 1 & $32,73^{* *}$ \\
\hline
\end{tabular}

** $=\mathrm{P}<0,01 ;{ }^{*}=\mathrm{P}<0,05 ; \mathrm{ns}=\mathrm{P}>0,05$. em que: $\hat{\mathrm{Y}}=$ peso ao nascer estimado; $\mathrm{X}=$ peso, em $\mathrm{kg}$, da búfala pós-parto.

O peso médio pós-parto da búfala e o desvio-padrão observados neste estudo foram de 538,70 $\pm 72,77 \mathrm{~kg}$. Schammass et al. (1996) relataram que o peso ao nascer depende do peso da fêmea após a cobrição. Soysal et al. (2005) afirmaram que esta característica é influenciada pelo peso corporal das fêmeas durante a gestação.

A herdabilidade estimada para peso ao nascer de bubalinos mestiços foi de $0,60 \pm 0,12$, um valor alto, que indica que esses animais provavelmente responderiam bem à seleção para o peso ao nascimento.

Outros valores de alta, moderada e baixa magnitude foram observados em trabalhos envolvendo herdabilidade para o peso ao nascer em bubalinos de diferentes raças. Cassiano et al. (2004), por exemplo, observaram herdabilidade de 0,62 para a raça Murrah e valores de 0,39; 0,28 e 0,16, respectivamente, para as raças Carabao, Jafarabadi e Mediterrânea. Balieiro et al. (1996), em rebanho com animais das raças Murrah, Jafarabadi e Mediterrânea, observaram herdabilidade de 0,10 $\pm 0,07$, indicando que, neste caso, a maior parte da variação é conseqüência de fatores ambientais.

Esses resultados comprovam a importância de fatores de herança sobre características produtivas, como peso ao nascer, e sugerem que esta característica pode ser utilizada na seleção precoce de bubalinos jovens criados sob semelhantes condições ambientais.

\section{Conclusões}

A idade ao primeiro parto é influenciada pelo grupo de contemporâneos da búfala ao nascimento, que deve ser considerado na avaliação da característica reprodutiva. Os efeitos linear e quadrático da idade ao parto anterior, o peso da búfala pós-parto anterior e o grupo de contemporâneos da búfala ao parto anterior devem ser considerados na avaliação do intervalo de partos de búfalas mestiças. Sexo do bezerro, grupo de contemporâneos do bezerro e peso da búfala após o parto devem ser considerados na avaliação da característica produtiva peso ao nascimento. Fêmeas mais pesadas produzem proles mais pesadas. A utilização da característica peso ao nascer de búfalos mestiços é recomendável em trabalhos de seleção.

\section{Agradecimento}

Ao Centro de Pesquisa Agroflorestal da Embrapa de Rondônia(CPAFRO/EMBRAPA-RO), pela disponibilização dos dados avaliados. 


\section{Literatura Citada}

ABDALLA, E.B. Improving the reproductive performance of Egyptian buffalo cows by changing the management system. Animal Reproduction Science, v.75, p.1-8, 2003.

AZIZ, M.A.; SCHOEMAN, S.J.; JORDAAN, G.F. et al. Genetic and phenotypic variation of some reproductive traits in Egyptian buffalo. South African Jornal of Animal Science, v.31, n.3, p.195-199, 2001.

BALIEIRO, E.S.; BALIEIRO, J.C.C.; VALENTE, J. Fatores de meio e herança como causas de variação no peso ao nascimento de bubalinos (Bubalus bubalis, L.). In: REUNIÃO ANUAL DA SOCIEDADE BRASILEIRA DE ZOOTECNIA, 33., 1996, Fortaleza. Anais... Fortaleza: Sociedade Brasileira de Zootecnia, 1996. p.296-297.

BARUSELLI, P.S.; OLIVEIRA, J.F.S.; MENDES, M.L.M. et al. Diagnóstico da bubalinocultura do Vale do Ribeira. Campinas: Coordenadoria de Assistência Técnica Integral, 1993. v.94, 16p. (DocumentoTécnico).

BARUSELLI, P.S.; HENRIQUEZ, C.E.P. [2002]. Reprodução em búfalos. Disponível em: <http://www2.fmvz.usp.br/bufalos/ index.html> Acesso em: 5/10/2007.

CASSIANO, L.A.P.; MARIANTE, A.S.; McMANUS, C. et al. Caracterização fenotípica de raças bubalinas nacionais e do tipo Baio. Pesquisa Agropecuária Brasileira, v.38, n.11, p.13371342, 2003.

CASSIANO, L.A.P.; MARIANTE, A.S.; McMANUS, C. et al. Parâmetros genéticos das características produtivas e reprodutivas de búfalos na Amazônia brasileira. Pesquisa Agropecuária Brasileira, v.39, n.5, p.451-457, 2004.

COSTA, C.N. Interação genótipo e ambiente em gado de leite. In: SIMPÓSIO INTERNACIONAL DE GENÉTICA E MELHORAMENTO ANIMAL, 1999, Viçosa, MG. Anais... Viçosa, MG: Universidade Federal de Viçosa, 1999. 426p.

DAS, A.; DAS, D.; GOSWAMI, R. et al. Growth performance of swamp buffaloes of Assam from birth to 12 months of age. Buffalo Buletin, v.23, n.4. p.84-89, 2004.

FAOSTAT data. [2005]. Disponível em: <http://faostat.fao.org/ faostat/servlet $>$. Acesso em: 20/1/2006.

INSTITUTO NACIONAL DE METEOROLOGIA - INMET [2006]. Disponível em: <http://www.inmet.gov.br/climatologia/combo _climatologia _I.html>. Acesso em: 15/1/2006.

MEYER, K. DFREML. [2000]. - Version 3.0 ß - user notes. Disponível em: <http://agbu.une.edu.au/ kmeyer//dfreml.html>. Acesso em: 13/7/2000.

NOGUEIRA, J.R.; CARDOSO, V.L.; MATTOS, J.C.A. et al. Sistema de produção de bubalinos para carne em pastagens de capim colonião na região noroeste do Estado de São Paulo. Boletim de Indústria Animal, v.54, n.1, p.25-32, 1997.
PEREIRA, J.C.C. Melhoramento genético aplicado à produção animal. 4.ed. Belo Horizonte: FEPMVZ, 2004. 609p.

RAMOS, A.A.; WECHSLER, F.S.; ONSELEN, V.J.V. et al. Sumário de touros bubalinos. Botucatu: UNESP/FMVZ, 2004. n.2, 37p. (PROMEBUL Boletim Técnico)

RIBEIRO, H.F.L.; ANDRADE, V.J.; MARQUES JR., A.P. et al. Efeito da condição corporal no momento do parto sobre o aparecimento do primeiro cio pós-parto em búfalas. Revista Brasileira de Medicina Veterinária, v.19, n.5, p.213-218. 1997.

SAMPAIO NETO, J.C.; MARTINS FILHO, R.; LÔBO, N.R.B. et al. Avaliação dos desempenhos produtivo e reprodutivo de um rebanho bubalino no Estado do Ceará. Revista Brasileira de Zootecnia, v.30, n.2. p.368-373, 2001.

STATISTICAL ANALYIS SYSTEM - SAS. SAS/STAT: user's guide. Version 6.12. 4.ed. v.2. Cary: SAS Institute, 2000. (CD-ROM).

SHAH, S.N.H. Influence of calving season and parity on resumption of postpartum ovarian cyclicity as assessed by milk progesterone profiles in Nili-Ravi buffaloes. In: WORLD BUFFALO CONGRESS, 3., 1991, Varnas, Bulgária. Proceedings... Varnas: Agricultural Academy, 1991. v.1.

SCHAMMASS, E.A.; KAWATOKU, M.; BARBOSA, C. et al. Desenvolvimento ponderal de bubalinos da raça mediterrânea. In: REUNIÃO ANUAL DA SOCIEDADE BRASILEIRA DE ZooteCniA, 33., 1996, Fortaleza. Anais... Fortaleza: Sociedade Brasileira de Zootecnia, 1996. p.301-303.

SILVA, M.E.T.; PEROTTO, D.; PINTO, J.M. et al. Desempenho de um sistema de búfalos da raça Murrah na região Nordeste do Paraná. Londrina: IAPAR, 1995. 24p. (Boletim Técnico, 49).

SOYSAL, M.I.; TUNA, Y.T.; GÜRCAN, E.K. An investigation on the water buffalo breeding in Danamandira Village of Silivri District of Istanbul Province of Turkey. Journal of Ttekirdag Agricultural Faculty, v.2, n.1, p.73-78, 2005.

TÉLLEZ, M.B.; MELÉNDEZ, Y.A.; MARTINEZ, A.M. et al. [2005]. Influencia de la época y región en algunos indicadores reproductivos del búfalo de agua (Bubalus bubalis) en el territorio oriental de Cuba. Revista Electrónica de Veterinaria, v.6, n.9. p.1-6. 2005. Disponível em: <http://www.veterinaria.org/ revistas/redvet/n090905.html> Acesso em: 5/10/2005.

TONHATI, H. Melhoramento. In: OLIVEIRA, G.J.C.; ALMEIDA, A.M.L.; SOUZA FILHO, U.A. (Eds.) O búfalo no Brasil. Cruz das Almas: Universidade Federal da Bahia, 1997. p.101-113.

TONHATI, H.; VASCONCELLOS, F.B.; ALBUQUERQUE, L.G. Genetics aspects of productive and reproductive traits in a Murrah buffalo herd in São Paulo, Brazil. Journal Animal Breeding Genetic, v.117, p.331- 336, 2000.

WOLFINGER, R.; O’CONNELL, M. Generalized linear mixed models: a pseudo-likelihood approach. Journal Statistical Computation and Simulation, v.48, p.233-243, 1993.

YADAV, B.S.; YADAV, M.C.; SINGH, A. et al. Murrah buffaloes. I. Birth weight. Buffalo Bulletin, v.20, n.2, p.29-31, 2001. 